\title{
Histone modification pattern evolution after yeast gene duplication
}

Yangyun Zou', Zhixi Su', Wei Huang ${ }^{2}$ and Xun Gu${ }^{1,2^{*}}$

\begin{abstract}
Background: Gene duplication and subsequent functional divergence especially expression divergence have been widely considered as main sources for evolutionary innovations. Many studies evidenced that genetic regulatory network evolved rapidly shortly after gene duplication, thus leading to accelerated expression divergence and diversification. However, little is known whether epigenetic factors have mediated the evolution of expression regulation since gene duplication. In this study, we conducted detailed analyses on yeast histone modification (HM), the major epigenetics type in this organism, as well as other available functional genomics data to address this issue.

Results: Duplicate genes, on average, share more common HM-code patterns than random singleton pairs in their promoters and open reading frames (ORF). Though HM-code divergence between duplicates in both promoter and ORF regions increase with their sequence divergence, the HM-code in ORF region evolves slower than that in promoter region, probably owing to the functional constraints imposed on protein sequences. After excluding the confounding effect of sequence divergence (or evolutionary time), we found the evidence supporting the notion that in yeast, the HM-code may co-evolve with cis- and trans-regulatory factors. Moreover, we observed that deletion of some yeast HM-related enzymes increases the expression divergence between duplicate genes, yet the effect is lower than the case of transcription factor (TF) deletion or environmental stresses.

Conclusions: Our analyses demonstrate that after gene duplication, yeast histone modification profile between duplicates diverged with evolutionary time, similar to genetic regulatory elements. Moreover, we found the evidence of the co-evolution between genetic and epigenetic elements since gene duplication, together contributing to the expression divergence between duplicate genes.
\end{abstract}

Keywords: Histone modification, Histone modification code divergence, Gene duplication, Expression divergence, Epigenetic divergence, cis-regulation, trans-regulation

\section{Background}

Although gene duplication has been widely considered as the main source of evolutionary novelties [1-4], the issue of duplicate gene preservation remains a subject of hot debate, i.e., how duplicate copies can escape from being pseudogenized and then evolve from an initial state of complete redundancy to a steady-stable state that both functionally divergent copies are maintained by purifying selection. As one of plausible hypotheses, rapid expression divergence between duplicate genes

\footnotetext{
* Correspondence: xungufudan@gmail.com

${ }^{1}$ Ministry of Education Key Laboratory of Contemporary Anthropology and

Center for Evolutionary Biology, School of Life Sciences, Fudan University,

Shanghai 200433, China

${ }^{2}$ Department of Genetics, Development, and Cell Biology, lowa State University, Ames, IA 50011, USA
}

may be the first step that is fundamental for the preservation of redundant duplicates [3]. There are three recognized types involved in expression regulatory mechanism: (i) cis-regulation of transcription mediated by promoters, enhancers, silencers, etc.; (ii) trans-regulation mediated by regulatory proteins binding to cis elements, such as transcription factors (TF); and (iii) epigenetic regulation, such as DNA methylation, specific histone modification pattern of genes (histone code hypothesis). Many studies have addressed the effect of cis- and trans- regulatory mechanisms on the expression divergence after gene duplication, e.g., cis-regulatory motif, TF-binding interaction, transacting expression quantitative trait loci (eQTLs) [5-10]. Though there is increasing evidence that epigenetic changes may play important roles in the initial expression divergence between duplicate genes [11-15], little study

\section{Biomed Central}


has been done about how regulatory network between duplicate genes evolve at epigenetic level.

Epigenetic regulation on gene expression is a highly complex process. In a broad sense, it includes DNA methylation, histone modification, nucleosome occupancy, as well as microRNA [11]. Moreover, these epigenetic elements can interact with each other, for instance, the reciprocity between DNA methylation and histone modification $[16,17]$. The complexity of epigenetic regulation has made it difficult to explore its role in regulatory divergence between duplicates. Nevertheless, we have recognized budding yeast (Saccharomyces cerevisiae) as an ideal organism for our purpose, because its epigenetic regulation is relatively simple: DNA cytosine methylation and microRNA were not detected $[18,19]$. In other words, histone modification is the main representation for epigenetic modification in the budding yeast, less affected by other epigenetic modification types. Therefore, in the present study, we focus on the evolution of histone modification (HM) between yeast duplicate genes.

Eukaryotic DNA with a unit of $146 \mathrm{bp}$ wound around a histone octamer (two copies of each core histone H2A, $\mathrm{H} 2 \mathrm{~B}, \mathrm{H} 3, \mathrm{H} 4)$ is assembled into chromatin. Histone Nterminal tails are subject to multiple covalent posttranslational modifications, including lysine (K) acetylation, lysine or arginine (R) methylation, serine (S) phosphorylation, and so on $[20,21]$. Enormous possible combinations and interactions of histone modification types constitute histone code. The 'histone code' hypothesis claims that a specific pattern of hisone modification code can produce a specific effect on local chromatin structure, modulating DNA accessibility, and consequently regulating transcription and other DNA-based biological processes [20-24].

Our goal is to investigate the pattern of yeast histone modification (HM) code divergence between duplicates. Our hypothesis claims $(i)$ that, when a gene is duplicated, the gene-specific histone modification profile is also duplicated, so on average duplicate pairs tend to have a higher degree of HM code similarity than randomly selected singleton gene pairs; and (ii) that since duplication, HM-code profile between duplicate copies become divergent with evolutionary time. We test these two predictions by conducting genome-wide analyses, as well as genes involved in different biological functions. Moreover, we are particularly interested in whether genetic regulatory elements, including cis-motifs (such as TATA box) and transcription factors, and epigenetic HM-code profile co-diverge during the evolution since gene duplication. To this end, time-dependent confounding factors in both genetic and epigenetic factors need to be ruled out. Finally, the significance of our study for having a better understanding of regulatory evolution after gene duplication is discussed.

\section{Results}

Combinational interactions of histone-modifying enzymes (HATs, HDACs, HMTs, HDMs, etc.) to histone N-tail produce numerous types of post-translational modification of histones $(\mathrm{H} 2 \mathrm{~A}, \mathrm{H} 2 \mathrm{~B}, \mathrm{H} 3, \mathrm{H} 4)$, such as methylation, acetylation [25]. Moreover, the same modification site can be affected by different modifying enzymes, and vice versa, generating different histone modification combinations, like H3K4me2 and H3K4ac (dimethylation and acetylation in Lys4 of histone H3, respectively), or H4K8ac (acetylation in Lys8 of histone H4). In this study, histone modification (HM) code of a gene represents the combined profile of different HM sites, HM types and HM states in gene promoter and open reading frame (ORF) regions, respectively (see Methods). We believe that the HM code of a gene reveals the pattern of HM mediated regulatory network of that gene.

\section{Functional redundancy in histone modification (HM) between yeast duplicate genes}

Because of evolutionary relatedness, duplicate pairs may have a higher similarity of histone modification (HM) code than two randomly selected single-copy genes (singletons). To test this hypothesis, we compared the distance of HM code between duplicate pair and randomized singleton pair. To be simple we choose one minus Pearson's product-moment correlation coefficient, i.e., $D_{H M}=$ $1-r$, to define the distance of HM code. The larger the value of $D_{H M}$, the higher divergence of histone modification code between duplicate genes. Specifically, denote the distance of HM code associated in promoter and ORF regions by $D_{H M-P}$ and $D_{H M-O}$, respectively. Randomized pairs were selected from single copy genes with 10000 repeats. As expected, we observed that both $D_{H M-P}$ and $D_{H M-O}$ measures show a lower divergence degree of histone modification code between duplicate genes than that of randomized singleton pairs (Wilcoxon rank sum test: $P<10^{-15}$ for both cases; Figure $1 \mathrm{~A}$ ).

Generally speaking, local chromatin environment around genes is one of important components leading to different histone modification code of each gene. While chromatin environment differs in different chromosomes, duplicate genes locating in different chromosomes may be under different chromatin environment, possessing the chromosomespecific HM profile. Following this argument, we classified all yeast gene pairs (duplicate and randomized singleton pairs) under study into two groups: they are located in the same chromosome, or different chromosomes, and tested the relationship between histone modification pattern and chromosome condition. We observed that though the HM profile divergence is not significantly correlated with location distance of gene pairs in the same chromosome (Pearson's product-moment correlation: $r=0.06, P=0.09$ for promoter region and $r=0.02, P=0.45$ for ORF region), 

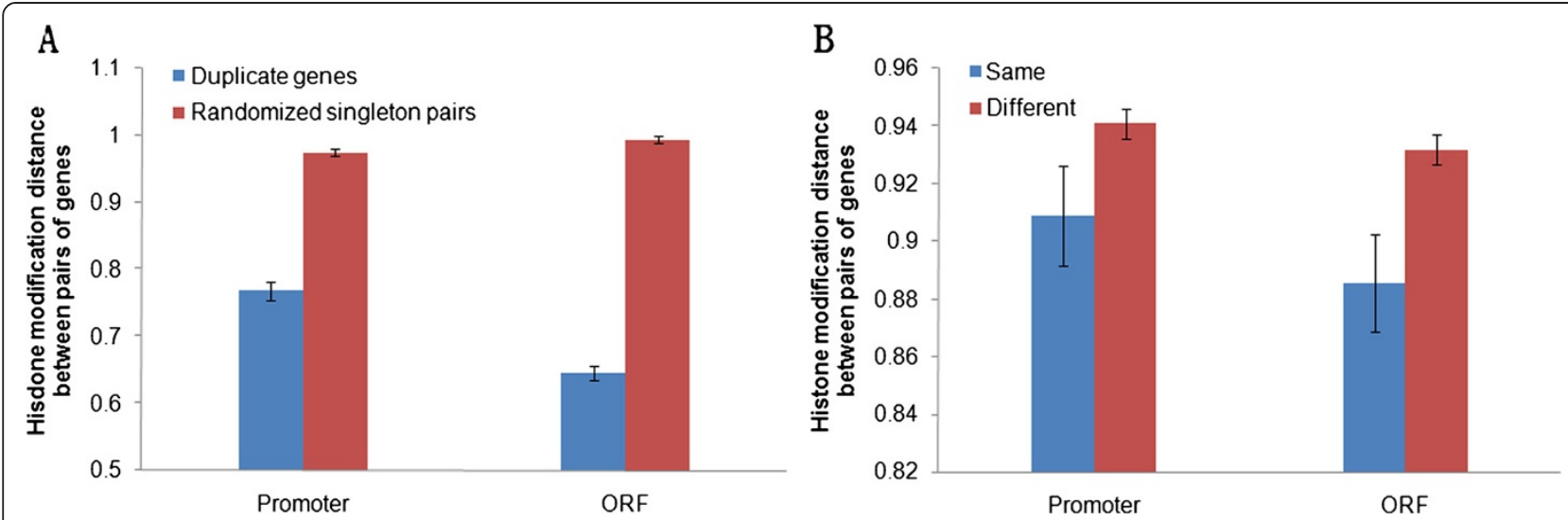

Figure 1 Comparison of the histone modification divergence between yeast genes. Comparison of the histone modification divergence between (A) duplicate genes and randomized pairs of single-copy genes, and (B) all pairs of genes in the same and different chromosomes.

gene pairs locating in the same chromosome share more common HM code than that in different chromosomes (Wilcoxon rank sum test: $P=0.07$ and $P=0.01$ for promoter and ORF regions, respectively; Figure 1B).

The chromosome effect on the HM-code divergence between duplicate genes may suggest an alternative interpretation about a higher similarity of HM distance between duplicate genes than random pairs. That is, duplicate pairs tend to be located in the same chromosome because of tandem gene duplications, compared to randomized singleton pairs (Chi-squared test: $\chi^{2}=17.2$, d.f. $=1, P<10^{-4}$ ). To rule out this possibility, we chose duplicate pairs and randomized singleton pairs where both copies are belonging to different chromosomes, and observed the similar result to Figure 1A (see Additional file 1: Figure S1). Hence, we conclude that duplicate genes represent their functional redundancy at the level of histone modification mediated regulatory network.

\section{The evolution of HM is coupled with coding sequence divergence after gene duplication}

We further expect that functional redundancy in histone modification code of duplicate genes as shown in Figure 1A would maintain a high degree in recently duplicated genes, and low in ancient duplicates. To verify this claim, we investigated the relationship between the distance of HM code $\left(D_{H M-P}\right.$ or $\left.D_{H M-O}\right)$ and coding sequence divergence (the synonymous distance $K_{S}$ or the nonsynonymous distance $K_{A}$ between duplicate genes). Considering the statistically unreliable estimation of synonymous or nonsynonymous substitution distance when $K_{S}$ or $K_{A}$ becomes larger because of repeated substitutions at the same site, we selected duplicate pairs with $K_{S}<2.0$ and $K_{A}<0.5$ for this analysis. We observed that both $D_{H M-P}$ and $D_{H M-O}$ are positively correlated with $K_{S}$ or $K_{A}$ (Pearson's product-moment correlation: all $r>0.45$, $P<10^{-15}$ for all data points; Figure 2), suggesting that the divergence of HM code is coupled with the coding sequence divergence between duplicate genes. To avoid correlated data points bringing the bias, we selected independent pairs of duplicate genes using the method from Zou et al. [9] and reanalyzed. The similar result remains hold (all $r>0.40, P<10^{-13}$ for all data points; in Additional file 1: Figure S2).

Considering $K_{S}$ or $K_{A}$ as a proxy to evolutionary time since gene duplication, we suggest that the correlation between the HM-code divergence and the coding sequence divergence has been mainly driven by mutations accumulated with evolutionary time. Our interpretation is based on two reasons: First, we observed a weak negative correlation between the HM divergence and the $K_{A} / K_{S}$ ratio of duplicate genes (Pearson's productmoment correlation: $r=-0.12, P<10^{-7}$ for $D_{H M-P}$ and $r=-0.05, P=0.04$ for $\left.D_{H M-O}\right)$. As the $K_{A} / K_{S}$ ratio is an indicator of sequence conservation in coding region, our finding implies that duplicate genes with stringent functional constraints on coding sequence may have greater divergence in the HM code, but the effect is marginal. Second, promoter HM code of duplicate genes diverges much quicker than that of ORF HM code (Wilcoxon rank sum test: $P<10^{-11}$; Figure 2), while significant but weak difference of $D_{H M-P}$ and $D_{H M-O}$ in randomized singleton pairs (Wilcoxon rank sum test: $P=0.02$; Figure 1 ). Some factors may be involved to accelerate the divergence of promoter HM code between duplicate genes, such as the evolution of transcription factors (TF) shared by duplicate genes.

\section{Co-evolution of the HM-code divergence between duplicates with several genetic regulatory elements}

The interaction between epigenetic and genetic elements in gene regulation has been increasingly acknowledged $[21,26]$, raising an interesting question whether the divergence of histone modification code between duplicate genes co-evolves with some trans-acting factors binding 

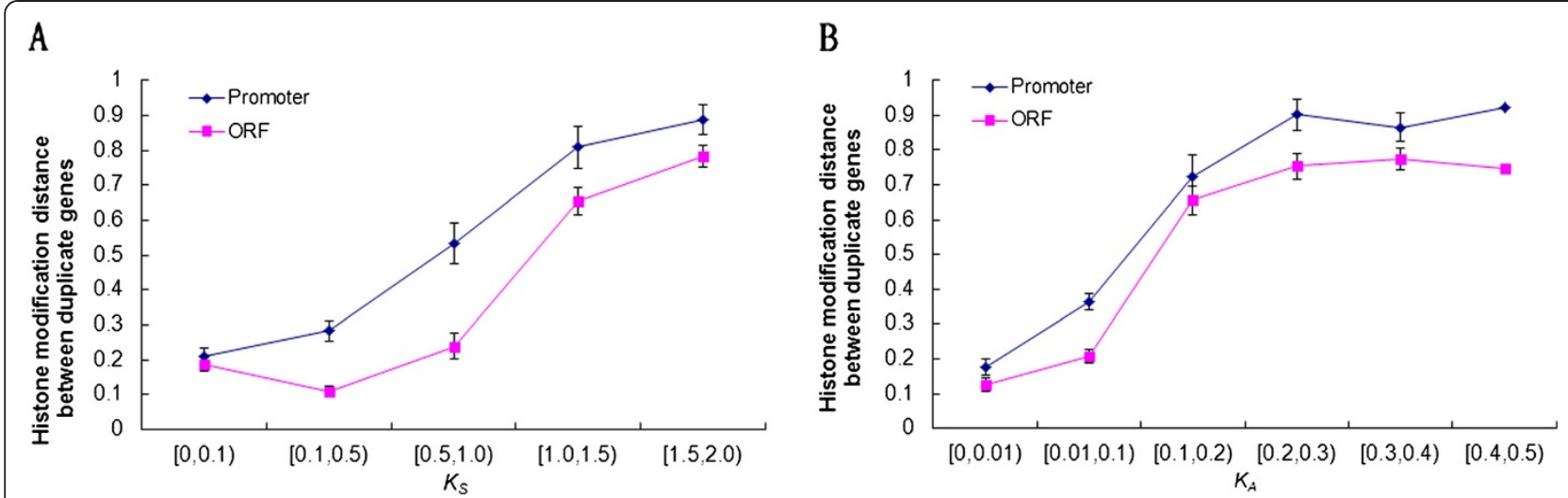

Figure 2 The divergence of histone modification pattern between duplicate genes $\left(D_{H M-P}, D_{H M-O}\right)$ increases with synonymous distance $K_{S}$ (panel A) or nonsynonymous distance $K_{A}$ (panel B) of duplicate genes.

to those duplicate genes. We first studied the relationship between the distance of promoter HM code $\left(D_{H M-P}\right)$ and the distance of transcription factors $\left(D_{T F}\right)$ and trans-acting expression quantitative trait loci (eQTLs) $\left(D_{t-e Q T L}\right)$ between duplicate genes. Trans-acting eQTLs of one gene represent all trans-regulatory proteins for its transcription, not limited to transcription factors. Two distance measures $D_{T F}$ and $D_{t-e Q T L}$ were determined by CzekanowskiDice formula (Methods). We found that they are significantly correlated (Peasron's product-moment correlation: $r>0.25, P<10^{-13}$; Figure 3A, 3C). Similar results were obtained in the case of ORF HM code (Figure 3B, 3D).

As both $D_{H M-P}$ and $D_{H M-O}$, as well as $D_{t-e Q T L}$ and $D_{T H}$ increase with evolutionary time ( $K_{S}$ or $K_{A}$ as the proxy) [Figure 2; 9], it is reasonable to suspect that $K_{S}$ or $K_{A}$ may underlie these statistically significant correlations between the HM code and genetic regulatory elements. We conducted the partial correlation in $D_{H M-P^{-}} D_{T F}$ and $D_{H M-P^{-}}$ $D_{t-Q Q T L}$ of duplicate genes under the controlling of $K_{S}$ and $K_{A}$ variables (with the restriction of $K_{S}<2.0$ and $K_{A}$ $<0.5$ ), and still observed the significant relationship $\left(r=0.18, P<0.001\right.$ for $D_{H M-P}-D_{T F}$ and $r=0.15, P<0.05$ for $\left.D_{H M-P}-D_{t-e Q T L}\right)$, though they are relatively weak. In short, our analysis provides the evidence that the histone modification code and trans-regulators shared by duplicate genes may have co-evolved since gene duplication.

Moreover, we design the following analysis to further explore the co-evolution between the HM code and transregulators (TF or trans-acting eQTL), by dividing yeast genes into two categories, trans-targeted genes and controlling genes. Trans-targeted genes are genes that are targeted by transcription factors (TF-targeted genes) or have at least one trans-acting eQTL (trans-eQTL acting genes) and the rest of genes are controlling genes (Methods). We totally obtained 4495 trans-targeted genes and 2226 controlling genes (Figure 4A). Interestingly, both promoter and ORF HM code distances of duplicates in the group of trans-targeted genes are, on average, significantly higher than those in the group of controlling genes (Figure 4B) (Wilcoxon rank sum test: promoter, $P=0.003$ and ORF, $P=0.0001$ ). It should be noticed that the pattern we observed in Figure 4B would not be affected by the strong correlation between the HM divergence and evolutionary time ( $K_{S}$ as the proxy) of duplicate genes, because the distribution of $K_{S}$ has been found no significant difference between trans-targeted genes and controlling genes (Wilcoxon rank sum test: $P=0.08$ ).

\section{The HM-code divergence and TATA-box regulation}

TATA box is the core promoter element for gene regulation responding to environmental stresses $[27,28]$. To examine the role of TATA box in the HM-code divergence between duplicate genes, we divided all yeast duplicate pairs into three groups, TATA-containing (both have TATA-box), TATA-less (both do not have TATA-box), and TATA_diverge (only one copy has TATA-box). We compared the HM-code distance in promoter $\left(D_{H M-P}\right)$ and ORF $\left(D_{H M-O}\right)$ region between duplicate genes in these groups. Interestingly, both $D_{H M-P}$ and $D_{H M-O}$ show the highest degree in the TATA-diverge group, and the lowest in the TATA-containing group (Figure 5) (Wilcoxon rank sum test: promoter, $P<10^{-10}$; ORF, $P<10^{-15}$ ).

Our explanation is as follows. Note that TATAcontaining genes may interact with some specific chromatin modification factors to regulate gene expression [29]. In the TATA_diverge group, only one duplicate with TATA-box has such interaction, resulting in a higher HM-code divergence between them. By contrast, in the case of TATA-containing group, both duplicates with TATA-box have similar interactions, resulting in a higher HM-code similarity between them.

\section{Biological functions and the HM-code divergence between duplicate genes}

Do different biological functions affect the level of the HM-code divergence between duplicate genes? We used 


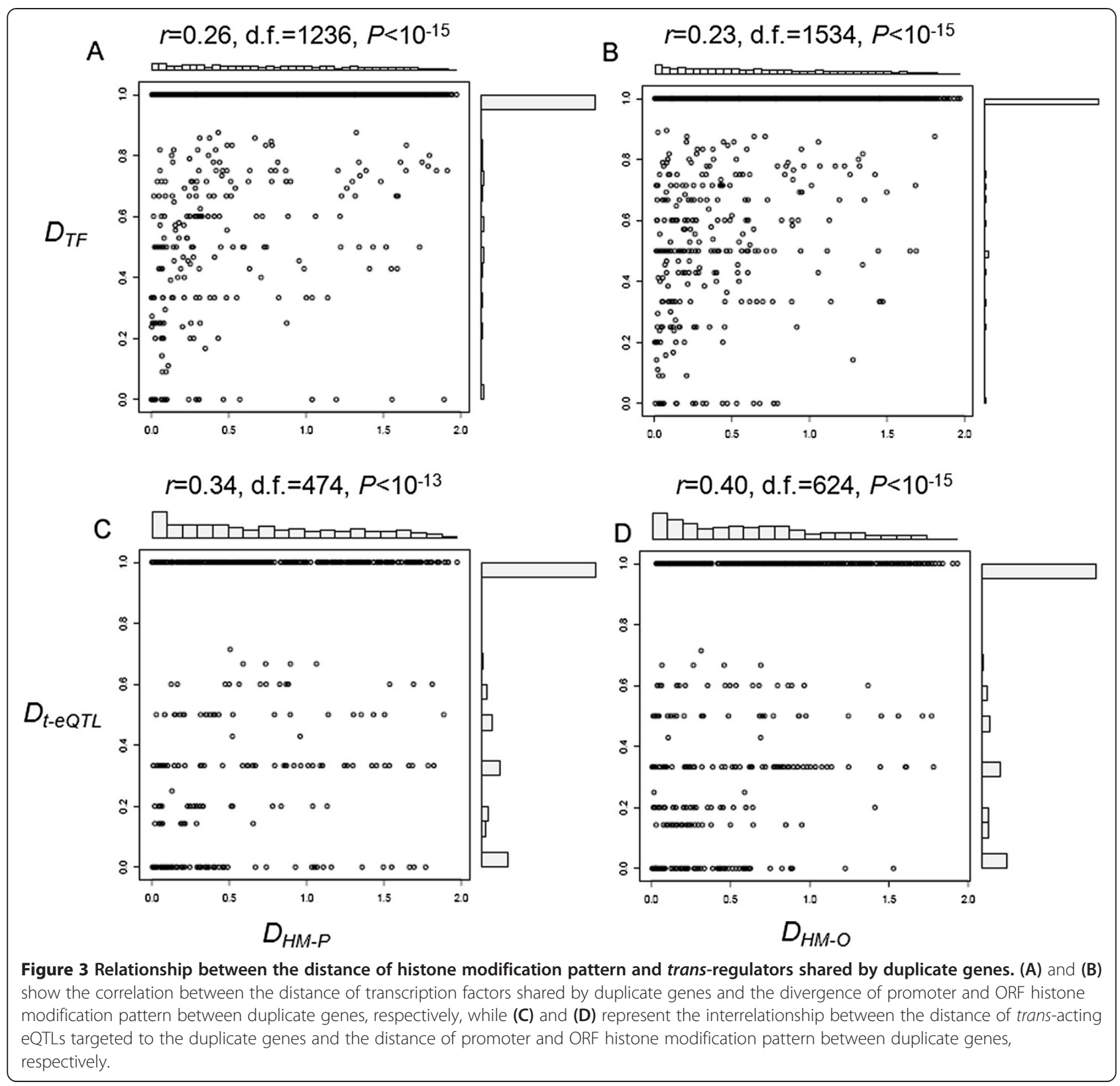

GO (Gene Ontology) Slim (biological process with 37 categories; see Methods) to address the issue. We analyzed $D_{H M-P}$ and $D_{H M-O}$ of duplicate genes in each GO category and compared $D_{H M-P}$ and $D_{H M-O}$ among these GO groups by one-way analysis of variance (ANOVA). Our finding is that duplicate pairs with different biological processes differ significantly in $D_{H M-P}$ and $D_{H M-O}\left(P<10^{-13}\right.$ for $D_{H M-P}$, $P<10^{-15}$ for $D_{H M-O}$; Table 1$)$. Use $D_{H M-P}$ for an example, duplicate genes involved in cofactor metabolic process, cellular amino acid and derivative metabolic process, cell cycle and cytoskeleton organization may have greater HM-code divergence, i.e., a higher $D_{H M-P}$, while duplicate genes involved in translation, DNA metabolic process, pseudohyphal growth and transcription may have lower divergence of the promoter HM code. Moreover, we conducted a similar analysis on 24 cellular components (GO Slim classification, see Methods), and observed that duplicate genes in different subcellular localization also undergo the different evolutionary rate of histone modification code after gene duplication (Table 2). It is possible that duplicate genes in some biological processes or subcellular localization are young (measured by small $K_{S}$ ) while others are old (with high $K_{S}$ ). Since $D_{H M-P}$ and $D_{H M-}$ $o$ are positively correlated with $K_{S}$ (Figure 2), we have to remove the confounding effect caused by $K_{S}$. We used the analysis of covariance (ANCOVA) ( $D_{H M-P}$ or $D_{H M-O} \sim$ 

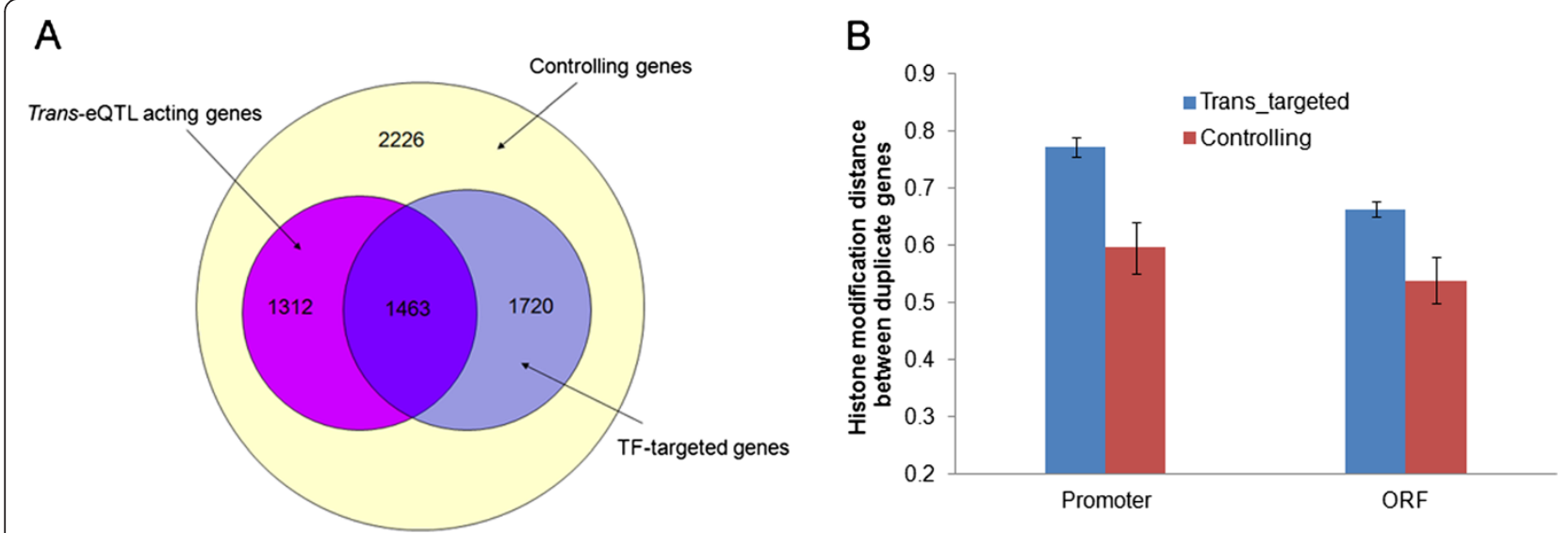

Figure 4 Different evolutionary rate of the histone modification code for duplicate genes in trans-targeted genes and controlling genes. (A) The information of different category genes, trans-targeted genes (TF-targeted genes and trans-eQTL acting genes) and controlling genes; (B) Comparison of the promoter histone modification distance $\left(D_{H M-P}\right)$ and the ORF histone modification distance $\left(D_{H M-O}\right)$ of duplicate genes between trans-targeted genes and controlling genes. Here, trans-targeted genes are the union of TF-targeted genes and trans-eQTL acting genes.

$K_{S}+\mathrm{T}$ (biological process or subcellular localization) $+K_{S}$ : $\mathrm{T})$ and the result remains statistically highly significant (Table 1, 2), suggesting $K_{S}$ is not a confounding factor that may affect our analyses.

The expression divergence under genetic, epigenetic and stressful perturbations

It is well-documented that gene expression divergence of duplicate genes increases with evolutionary time, but the underlying mechanism remains a subject of debate [30]. The analysis we describe below is to know whether the divergence of HM-mediated regulatory network affects the expression divergence between duplicate genes. We compared the interrelation between the histone modification pattern distance $\left(D_{H M-P}, D_{H M-O}\right)$ and the expression distance $(E)$ between duplicate genes, and found that they are significantly correlated (Pearson's

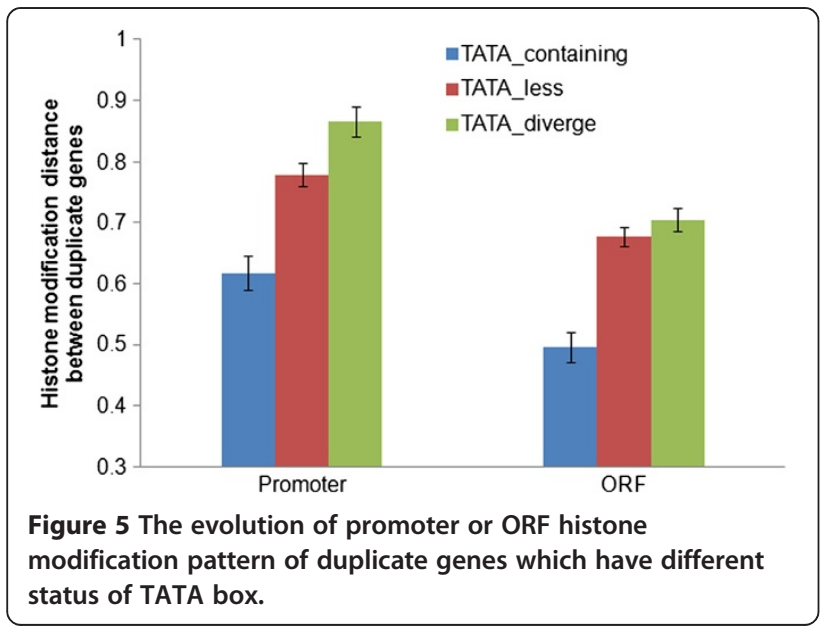

product-moment correlation; $D_{H M-P}-E: r=0.24, P<10^{-}$ ${ }^{15}$; $D_{H M-O}-E: r=0.30, P<10^{-15}$; Figure 6).

We further divided yeast expression profiles into four types from different perturbation conditions: 1) normal developmental or physiological conditions, defined as 'Normal' treatment; 2) a set of conditions where expression changes attribute to environmental stresses, denoted as 'Stress' treatment; 3) conditions where single gene coding chromatin modifiers (CM) like SWI/SNF, HDACs, HATs, etc. was deleted, denoted as 'CM_del' treatment; 4) conditions where single gene coding transcription factors (TF) was deleted, denoted as 'TF_del' treatment. The latter two types are able to test the effect of chromatin modification related proteins and transcription factors on other genes, respectively. We then calculated the expression divergence between duplicate genes under these four types of conditions, denoted by $E_{\text {Normal }}, E_{\text {Stress }}, E_{\mathrm{CM} \_ \text {del }}, E_{\mathrm{TF} \_ \text {del }}$, respectively. We observed that the expression distance between duplicate genes in 'CM_del' condition ( $E_{\mathrm{CM}}$ _del $)$ is significantly greater than that in 'Normal' condition ( $E_{\text {Normal }}$ ) (Wilcoxon rank sum test: $P<10^{-10}$; Figure $7 \mathrm{~A}$ ), but much lower than that in 'Stress' and 'TF_del' conditions $\left(E_{\text {Stress }}\right.$ and $\left.E_{\text {TF_del }}\right)$ (Wilcoxon sum rank test, $P<10^{-}$ ${ }^{15}$; Figure 7A). Results imply that histone modification related enzymes and gene associated histone modification profile may indeed influence the expression evolution of duplicate genes, though the relative contribution to the expression divergence between duplicate genes is highly lower than genetic related factors like transcription factors, even externally environmental stresses.

TATA-containing genes are usually enriched in stressrelated genes [29], and represent high expression variability $[31,32]$. In our study, we detected that in four disturbed 
Table 1 The divergence of histone modification code associated with promoter or ORF region of duplicate genes involving in $\mathbf{3 7}$ biological processes

\begin{tabular}{|c|c|c|c|c|}
\hline \multirow[t]{2}{*}{ Biological Process } & \multicolumn{2}{|c|}{$D_{H M-P}$} & \multicolumn{2}{|c|}{$D_{H M-O}$} \\
\hline & Mean & S.E. ${ }^{a}$ & Mean & S.E. \\
\hline Cofactor metabolic process & 1.089857 & 0.099487 & 0.94615 & 0.079585 \\
\hline Cellular amino acid and derivative metabolic process & 1.056124 & 0.075125 & 0.735075 & 0.058636 \\
\hline Cell cycle & 1.022464 & 0.070499 & 0.628888 & 0.045862 \\
\hline Cytoskeleton organization & 1.011731 & 0.100702 & 0.732692 & 0.068568 \\
\hline Carbohydrate metabolic process & 0.957513 & 0.041359 & 0.801785 & 0.03478 \\
\hline Cell wall organization & 0.943616 & 0.078784 & 0.919398 & 0.069325 \\
\hline Membrane organization & 0.943309 & 0.067702 & 0.786555 & 0.069908 \\
\hline Lipid metabolic process & 0.93571 & 0.079815 & 0.731593 & 0.059279 \\
\hline Generation of precursor metabolites and energy & 0.928247 & 0.096518 & 0.882262 & 0.072135 \\
\hline Anatomical structure morphogenesis & 0.921051 & 0.079036 & 0.777731 & 0.073299 \\
\hline Protein modification process & 0.900252 & 0.038873 & 0.854947 & 0.030103 \\
\hline Protein folding & 0.895056 & 0.060764 & 0.677196 & 0.05632 \\
\hline Response to stress & 0.891941 & 0.051014 & 0.766131 & 0.04367 \\
\hline Protein catabolic process & 0.877025 & 0.072138 & 0.74283 & 0.065929 \\
\hline Vesicle-mediated transport & 0.867052 & 0.045818 & 0.763747 & 0.04406 \\
\hline Cytokinesis & 0.855475 & 0.128721 & 0.458555 & 0.075571 \\
\hline Vitamin metabolic process & 0.844587 & 0.123271 & 0.697339 & 0.095067 \\
\hline Transport & 0.819455 & 0.026288 & 0.726211 & 0.021028 \\
\hline Heterocycle metabolic process & 0.812252 & 0.080271 & 0.737343 & 0.070763 \\
\hline Meiosis & 0.803064 & 0.204095 & 0.586892 & 0.16534 \\
\hline Cell budding & 0.798157 & 0.088983 & 0.814543 & 0.12039 \\
\hline Signal transduction & 0.796985 & 0.069456 & 0.846847 & 0.05721 \\
\hline RNA metabolic process & 0.792216 & 0.043033 & 0.562501 & 0.032646 \\
\hline Cellular aromatic compound metabolic process & 0.783433 & 0.116406 & 0.685336 & 0.092675 \\
\hline Ribosome biogenesis & 0.745581 & 0.078664 & 0.489313 & 0.057953 \\
\hline Cellular respiration & 0.741278 & 0.175087 & 0.970903 & 0.130576 \\
\hline Conjugation & 0.741105 & 0.115964 & 0.561448 & 0.065045 \\
\hline Cellular homeostasis & 0.732899 & 0.107582 & 0.622864 & 0.080052 \\
\hline Sporulation resulting in formation of a cellular spore & 0.726966 & 0.119265 & 0.430464 & 0.083954 \\
\hline Organelle organization & 0.709827 & 0.043332 & 0.569849 & 0.029456 \\
\hline Response to chemical stimulus & 0.675718 & 0.055068 & 0.590837 & 0.045851 \\
\hline Nucleus organization & 0.668734 & 0.212327 & 0.396937 & 0.134765 \\
\hline Transcription & 0.586723 & 0.061277 & 0.490756 & 0.047904 \\
\hline Pseudohyphal growth & 0.523636 & 0.183077 & 0.741094 & 0.154572 \\
\hline DNA metabolic process & 0.520462 & 0.057569 & 0.403555 & 0.03735 \\
\hline Translation & 0.475206 & 0.058331 & 0.32502 & 0.050416 \\
\hline Other & 0.803085 & 0.04149 & 0.791027 & 0.036751 \\
\hline ANOVA test & $F=3.91, P$ & & $F=7.60, P$ & \\
\hline ANCOVA test & $F=2.24, P$ & & $F=4.78, P$ & \\
\hline
\end{tabular}

${ }^{a}$ : S.E. denotes the standard error. 
Table 2 The divergence of histone modification code associated with promoter or ORF region of duplicate genes involving in 24 cellular components

\begin{tabular}{|c|c|c|c|c|}
\hline \multirow[t]{2}{*}{ Cellular Component } & \multicolumn{2}{|c|}{$D_{H M-P}$} & \multicolumn{2}{|c|}{$D_{H M-O}$} \\
\hline & Mean & S.E. & Mean & S.E. \\
\hline Cytoplasm & 0.815386 & 0.022075 & 0.691933 & 0.018111 \\
\hline Mitochondrion & 0.804229 & 0.045602 & 0.775065 & 0.037205 \\
\hline Ribosome & 0.401566 & 0.051071 & 0.21871 & 0.044488 \\
\hline Nucleus & 0.757928 & 0.03278 & 0.633467 & 0.025154 \\
\hline Membrane & 0.867299 & 0.045905 & 0.719912 & 0.037068 \\
\hline Mitochondrial envelope & 0.850156 & 0.071138 & 0.845782 & 0.058631 \\
\hline Nucleolus & 1.041006 & 0.088386 & 0.885202 & 0.076221 \\
\hline Chromosome & 0.848459 & 0.121672 & 0.500084 & 0.069019 \\
\hline Membrane fraction & 0.728517 & 0.057793 & 0.49282 & 0.052274 \\
\hline Cell wall & 0.703015 & 0.05532 & 0.397484 & 0.060315 \\
\hline Vacuole & 0.560115 & 0.090545 & 0.725808 & 0.070671 \\
\hline Golgi apparatus & 1.020564 & 0.07273 & 0.856226 & 0.060742 \\
\hline Cytoplasmic membrane-bounded vesicle & 0.780526 & 0.149402 & 0.703323 & 0.11963 \\
\hline Endomembrane system & 0.77575 & 0.132578 & 0.712372 & 0.098021 \\
\hline Cellular bud & 0.877352 & 0.094858 & 0.65631 & 0.069712 \\
\hline Cytoskeleton & 0.883771 & 0.094073 & 0.589399 & 0.07159 \\
\hline Microtubule organizing center & 0.664255 & 0.193299 & 0.553038 & 0.177284 \\
\hline Site of polarized growth & 0.875764 & 0.0889 & 0.636855 & 0.064332 \\
\hline Endoplasmic reticulum & 0.877175 & 0.081264 & 0.673596 & 0.056244 \\
\hline Plasma membrane & 0.72374 & 0.034621 & 0.70187 & 0.027569 \\
\hline Peroxisome & 0.500936 & 0.245856 & 0.827018 & 0.120535 \\
\hline Extracellular region & 0.553479 & 0.167985 & 0.224751 & 0.129525 \\
\hline Cell cortex & 1.00632 & 0.081755 & 0.650496 & 0.076325 \\
\hline Other & 0.752484 & 0.047213 & 0.632212 & 0.038696 \\
\hline ANOVA test & \multicolumn{2}{|c|}{$F=3.54, P<10^{-7}$} & \multicolumn{2}{|c|}{$F=6.53, P<10^{-15}$} \\
\hline ANCOVA test & \multicolumn{2}{|c|}{$F=1.77, P=0.01$} & \multicolumn{2}{|c|}{$F=4.43, P<10^{-10}$} \\
\hline
\end{tabular}

conditions (Normal, Stress, CM-del, TF_del), the expression divergence in TATA-diverge and TATA-containing groups are significantly larger than that in TATA-less duplicate genes (Figure 7B). The discrepancies between expression change and the histone modification divergence in these duplicate gene types (TATA-containing, TATA-less, TATAdiverge) are observed.

\section{Discussion}

The divergence of HM profile between duplicate genes

Our detailed analyses on yeast whole-genome histone modification (HM) code profile have shown that duplicate genes share more common HM-code patterns than randomized singleton pairs in their promoter and ORF regions, and the HM-code divergence between duplicates in both regions increase with the sequence divergence. This finding supports the notation that epigenetic divergence between duplicate genes may have been driven by the accumulation of mutations with the duplication time in both their promoter and ORF regions, because it has been shown that the divergence of coding sequence such as $K_{S}$ between yeast duplicates is a proxy to evolutionary time. In other words, no external driving force is needed to explain the HM profile divergence between duplicates, though it remains possible of neofunctionalization based upon divergent $H M$ profile through some positive selection mechanisms.

\section{Hypothesis-based genomic correlation analysis}

Genome-wide functional analysis of duplicate genes is in attempt to reveal functional correlation between genetic and epigenetic factors during the process of functional innovation through gene duplication. However, the universal confounding effect of the sequence divergence 
A

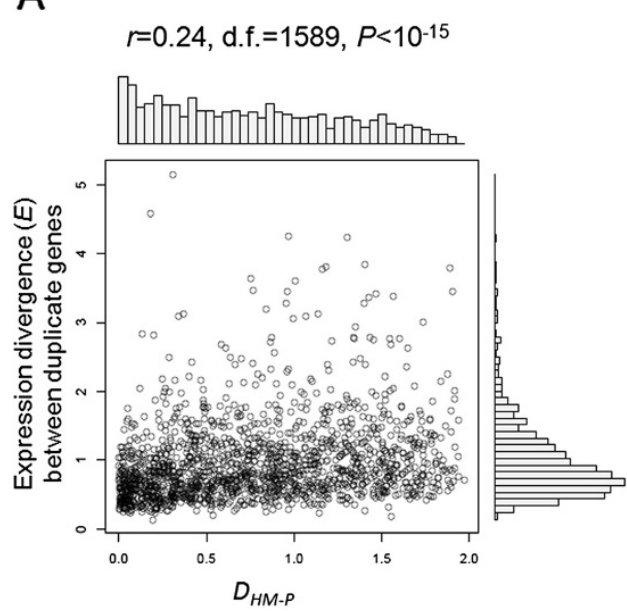

B

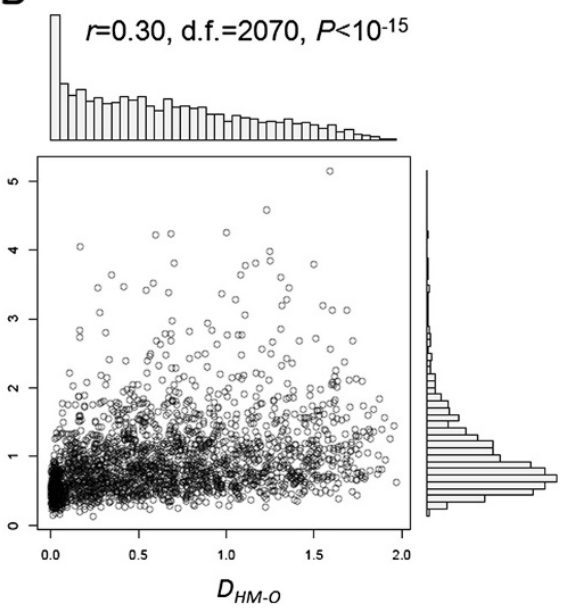

Figure 6 Correlation between the distance of histone modification pattern and the expression divergence $(E)$ of duplicate genes.

Relationship between the expression distance and $(\mathbf{A})$ the promoter histone modification divergence $\left(D_{H M-P}\right)$ and $(\mathbf{B})$ the ORF histone

modification divergence $\left(D_{H M-O}\right)$.

$\left(K_{S}\right)$ has complicated the practical analyses. Consequently, the controversy about the cause-effect interpretation has been inevitable, because genome-wide analysis of duplicate genes has been viewed as an exploration rather than a hypothesis-testing approach.

Since the correlation between the HM profile divergence and the sequence divergence actually reflects the fundamental evolutionary process driven by the accumulation of mutations, we view this as a null hypothesis in the genome-wide functional analysis of duplicate genes. That is, any meaningful inference about the functional correlation within or between genetic and epigenetic elements needs to reject this null hypothesis, as we have shown in this study.
Interaction between genetic and epigenetic elements: who is the driver?

The interaction between epigenetic and genetic elements in gene regulation has been increasingly acknowledged. For instance, the establishment of the histone modification code may partially involve the recruitment of specific histone modifying enzymes such as HATs, HDACs, HMTs by transcription factors (TF) [26]. Meanwhile, the distinctly combinatory histone modification code associated with gene may also provide specific binding code that is read by other transcription factors [21]. These observations raise an interesting question whether the divergence of histone modification code between duplicate genes may co-evolve with trans-acting factors binding to those
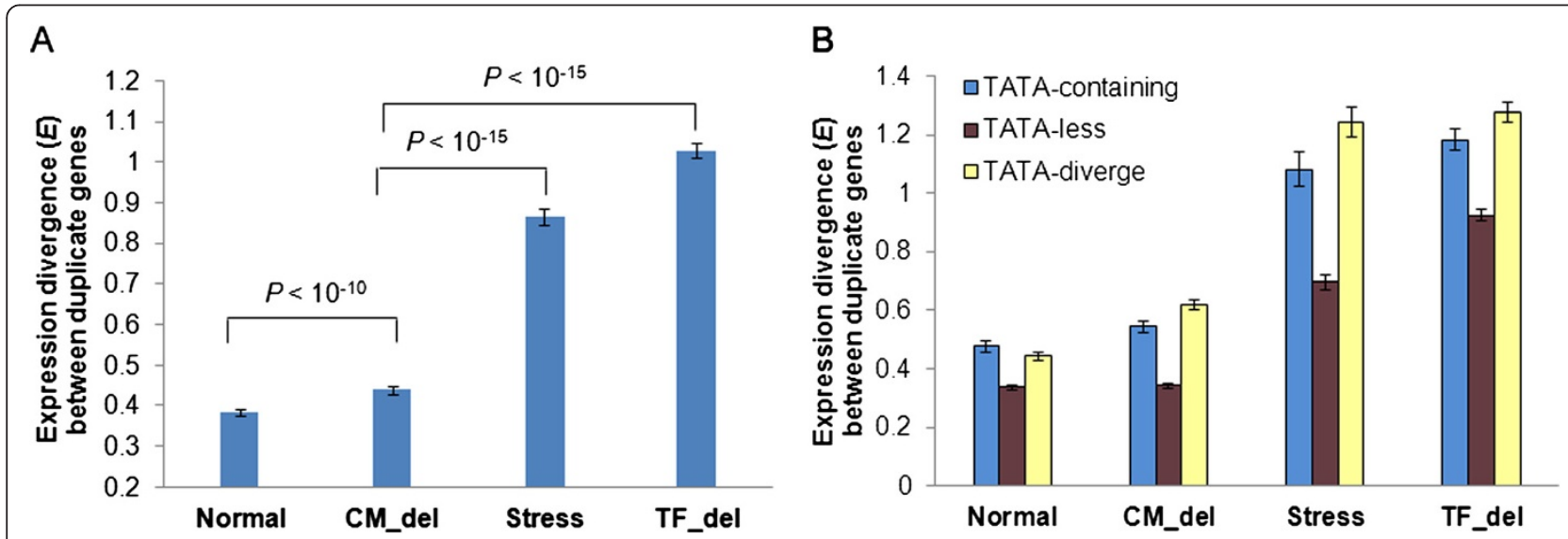

Figure 7 Comparison of the expression divergence between duplicate genes under different disturbing conditions. (A) The expression divergence between all duplicate genes; (B) The expression divergence between duplicate genes with different status of TATA box. P-value was obtained from Wilcoxon rank sum test using the open-source R statistical analysis language. 
duplicate genes, e.g., transcription factors, histone modifying enzymes.

We have observed a higher divergence for HM code of duplicate genes in the category of trans-targeted genes than that of controlling genes, suggesting that the change of trans-regulators binding to duplicate genes may affect the pattern of HM code in both promoter and ORF regions, and thus accelerating the divergence of histone modification code between duplicate genes. Yet, it remains unclear about the cause-effect relationship. For instance, does the divergence of trans-regulators to duplicate genes facilitate the divergence of histone modification between duplicate genes, or vice versa? Our further study will address this issue.

\section{Functional preference in the HM code divergence between duplicate genes}

We observed the functional bias on the HM-code divergence after gene duplication. One possibility is that histone proteins associated with genes in different biological functions may be subject to differentially post-translational modification, leading to different divergence rate of histone modification code between duplicate genes. Since histone modification process of one gene largely depends on its chromatin environment and DNA sequence interacted by histone modifying enzymes [33], functionally selective constraints may also be imposed on histone modification evolution associated with that gene, a situation similar to DNA sequence evolution.

\section{Conclusions}

In this study, we unveiled the evolution of yeast histone modification code since gene duplication. Though duplicate genes represent functional redundancy at histone modification level compared with single-copy genes, the histone modification divergence occurred along with evolutionary time ( $K_{S}$ as the proxy), which possibly due to the coding sequence evolution after gene duplication. Moreover, the histone modification code in ORF region evolves slower than that in promoter region, indicative of functionally selective constraints on protein sequences. Going further, after controlling the confounding effect of the coding sequence divergence $\left(K_{S}\right)$, the histone modification code co-evolves with cis- (TATA box) and trans- (TF and trans-acting eQTL) regulatory factors, confirmed by the fact that the histone modification code is shaped by the combined interaction among histone-modifying enzymes, trans-acting elements and cis-regulatory motif. In addition, histone modification makes contribution to the expression divergence between duplicate genes, despite the minor effect compared to transcription factors and environmental stresses. Taking together, we provided the evidence of the co-evolution between genetic and epigenetic elements since gene duplication, together contributing to the expression divergence between duplicate genes.

\section{Methods}

\section{Data of yeast histone modification pattern}

Genome-wide histone modification pattern data of Saccharomyces cerevisiae were downloaded from ChromatinDB [34] (http://www.bioinformatics2.wsu.edu/cgi-bin/ ChromatinDB/cgi/visualize_select.pl). ChromatinDB provides the user with easy access to ChIP-microarray data for a large set of histones or histone modifications in S. cerevisiae, which includes 17 distinct histone modification combinations like dimethylation in Lys4 of histone $\mathrm{H} 3$ (H3K4me2), acetylation in Lys12 of histone H4 (H4K12ac), etc. and 5 histone protein occupancy levels (H2A, H2B, H3, H4, H2A.Z). We applied log base-2 of average enrichment ratio with nucleosome-normalizing for each of 22 histone modification data in promoter and open reading frame (ORF) regions of genes.

\section{Yeast microarray expression data}

A total of 84 microarray expression data points of S. cerevisiae whose expression changes are attributing to internal disturbing like developmental or physiological conditions were respectively collected [35-37]. This type was defined as "Normal" conditions, which are not genetically perturbed by regulatory network related elements like transcription factors and chromatin modifiers $(\mathrm{CM})$ or other environmental stresses. We collected 170 gene expression profiles of yeast strains mutated for various chromatin modifiers from 26 publications [38]. We called this type of expression profile data as 'CM_del' conditions. Expression profile data of 263 transcription factor-deletion experiments were obtained from the Gene Expression Omnibus (GEO) database under the series accession number GSE4654 [39]. Similarly, this type was denoted as 'TF_del' experiments. A total of $504 \mathrm{cDNA}$ microarray data points of yeast whose expression changes are attributing to environmental stresses were collected [9]. We call this type as 'Stress' conditions. Normalization was done as each original paper recommended.

\section{Data of trans- and cis- regulatory elements}

Transcription factor (TF)-DNA binding profiles of yeast were downloaded from Lee et al. [40] and Harbison et al.[41]. In the study of Harbison et al. (2004), we just used 203 DNA-binding transcription factors in rich media conditions, regardless of 84 regulators in environmental stressed conditions. Most transcription factors in two studies are overlapped. Finally, we obtained 207 TFall S. cerevisiae genes binding interaction profiles. For each gene, a p-value was assigned to measure the probability of true TF-target interaction; a smaller p-value means the interaction is more likely. Here, we used 
relatively stringent significance level of 0.001 as cutoff to define the status of TF-target gene interaction. Two studies together determine all TF-target gene interactions, and we observed that 3183 genes are binding by at least one transcription factor (TF-targeted genes). Yeast genomic expression quantitative trait loci (eQTLs) data were downloaded from Brem and Kruglyak [42]. Wilcoxon-Mann-Whitney (WMW) test with the criterion of $50 \mathrm{~kb}$ interval was conducted to detect and define eQTL regions [9]. Finally, we obtained 2775 genes which at least have one trans-acting eQTL (trans-eQTL acting genes). Thus, we can divide all yeast genes into two categories, trans-targeted genes and controlling genes, where trans-targeted genes are the union of TF-targeted genes and trans-eQTL acting genes, while controlling genes are the reminders. Since trans-eQTL acting genes were not only regulated by transcription factors, but most by chromatin related enzymes and other factors [43], trans-targeted genes may have the possibility to be regulated by all trans-regulators, not restricted to transcription factors.

There are two types of genes, TATA-containing genes and TATA-less genes [29]. We classified all duplicate pairs into three categories, TATA-containing, TATA-less and TATA-diverge pairs. TATA-containing and TATA-less types are those duplicate pairs where both copies have or don't have TATA box, respectively, while TATA-diverge type refers to duplicate pairs where one copy belongs to TATA-containing genes, and the other TATA-less genes.

\section{Protein subcellular localization and biological process}

The information of protein localization and biological process for S. cerevisiae was defined by the Gene Ontology (GO) classification, and downloaded from Saccharomyces Genome Database. GO Slim was used to classify 24 cellular component sorts and 37 biological process categories. A duplicate gene pair was assigned to a GO Slim term if both duplicate copies are belonging to this GO term, or one copy is belonging to while the other is not annotated.

\section{Defining functional divergence between yeast duplicate genes}

There are two types of histone modification profiles, histone modification pattern associated with gene promoter region and open reading frame $(\mathrm{ORF})$ region. One minus Pearson's product-moment correlation coefficient (1-r) was used to determine the distance of these two types of histone modification pattern between duplicate genes, shortly denoted as $D_{H M-P}$ for promoter histone modification distance and $D_{H M-O}$ for ORF histone modification distance.

We modified the Czekanowski-Dice formula [44] to calculate the distance of transcription factors or trans-acting eQTLs shared by duplicate gene 1 and 2 in a duplicate pair, shortly denoted as $D_{T F}$ and $D_{t-e Q T L}$, respectively. Suppose $\Delta_{12}$ be the number of TFs or trans-acting eQTLs that differ between one duplicate pair; $y_{1} \cup y_{2}$ be the number of TFs or trans-acting eQTLs that regulate at least one of duplicate genes, and $y_{1} \cap y_{2}$ be the number of shared TFs or trans-acting eQTLs between a duplicate pair. Then, the TF distance or trans-acting eQTL distance between duplicate genes 1 and 2 is defined as follows:

$$
\begin{array}{r}
D_{T F}(1,2) \text { or } D_{t-e Q T L}(1,2) \\
=\Delta_{12} /\left[y_{1} \cup y_{2}+y_{1} \cap y_{2}\right]
\end{array}
$$

Apparently, the greater the value, the higher degree of TF or trans-acting eQTL divergence between duplicate genes.

We used evolutionary distance $(E)$ defined by $\mathrm{Gu}$ et al. [5] as the measure of expression divergence between duplicate genes of four types, shortly denoted as $E_{\mathrm{Normal}}$, $E_{\mathrm{CM} \text { _del }}, E_{\mathrm{TF} \text { _del }}$ and $E_{\text {Stress }}$ for 'Normal,' 'CM_del,' 'TF_del' and 'Stress' experiments, respectively. Specifically, for any duplicate gene 1 and 2 , let $x_{1 k}$ and $x_{2 k}$ be its expression level, respectively, in the $k$ th microarray experiment; $\bar{x}_{1}$ and $\bar{x}_{2}$ be the mean of expression level in $k$ th microarray experiments, respectively, where $k=1, \ldots m$. The formula of the expression distance $(E)$ between gene 1 and 2 is as follows:

$$
\hat{E}_{12}=\sum_{k=1}^{m}\left[\left(x_{1 k}-\bar{x}_{1}\right)-\left(x_{2 k}-\bar{x}_{2}\right)\right]^{2} /(m-1)
$$

\section{Determination of yeast duplicate pairs}

The method of Gu et al. [45] was applied to identify duplicate genes. As the criterion of $80 \%$ alignable regions between protein sequences is too stringent, and may miss some duplicate genes, we reduced this criterion to $50 \%$. All pairs of duplicate genes in each gene family were used for the analysis. The reminders of $S$. cerevisiae genes were considered as singleton genes. The rate of synonymous substitutions $\left(K_{S}\right)$ and nonsynonymous substitutions $\left(K_{A}\right)$ between duplicate genes were estimated using PAML [46] with default parameters.

\section{Additional file}

Additional file 1: Figures S1-S2. are available at online web site of BMC Evolutionary Biology journal.

\section{Abbreviations}

HATs: Histone acetyltransferases; HDACs: Histone deacetylases; HMTs: Histone methyltransferases; HDMs: Histone demethylases; $K_{S}$ : The rate of synonymous substitutions; $K_{A}$ : The rate of nonsynonymous substitutions; TF: Transcription factor; eQTLs: Expression quantitative trait loci; ORF: Open reading frame; HM: Hitone modification; ANOVA: Analysis of variance; ANCOVA: Analysis of covariance; GO: Gene ontology; CM: Chromatin modifiers. 


\section{Competing interests}

The authors declare that they have no competing interests.

\section{Acknowledgements}

We thank Libing Shen for his suggestion on English writing when we prepare the manuscript. This work was supported by the grant from the Ministry of Science and Technology China (2012CB910101 to ZS).

\section{Authors' contributions}

YZ designed and performed the study, analyzed the data and drafted the manuscript. ZS and WH analyzed the data. XG designed the study and drafted the manuscript. All authors read and approved the final version of the manuscript.

Received: 21 February 2012 Accepted: 9 July 2012

Published: 9 July 2012

\section{References}

1. Innan H, Kondrashov F: The evolution of gene duplications: classifying and distinguishing between models. Nat Rev Genet 2010, 11:97-108.

2. Lynch M, Force A: The probability of duplicate gene preservation by subfunctionalization. Genetics 2000, 154:459-473.

3. Ohno S: Evolution by gene duplication. Berlin: Springer; 1970.

4. Taylor JS, Raes J: DUPLICATION AND DIVERGENCE: The evolution of new genes and old ideas. Annu Rev Genet 2004, 38:615-643.

5. Gu X, Zhang Z, Huang W: Rapid evolution of expression and regulatory divergences after yeast gene duplication. Proc Natl Acad Sci U S A 2005, 102:707-712.

6. Leach $\sqcup$, Zhang Z, Lu C, Kearsey MJ, Luo Z: The role of cis-regulatory motifs and genetical control of expression in the divergence of yeast duplicate genes. Mol Biol Evol 2007, 24:2556-2565.

7. Papp B, Pal C, Hurst L: Evolution of cis-regulatory elements in duplicated genes of yeast. Trends Genet 2003, 19:417-422.

8. Zhang Z, Gu J, Gu X: How much expression divergence after yeast gene duplication could be explained by regulatory motif evolution? Trends Genet 2004, 20:403-407.

9. Zou Y, Su Z, Yang J, Zeng Y, Gu X: Uncovering genetic regulatory network divergence between duplicate genes using yeast eQTL landscape. J Exp Zool B Mol Dev Evol 2009, 312B:722-733.

10. Zou YY, Huang W, Gu ZL, Gu X: Predominant gain of promoter TATA box after gene duplication associated with stress responses. Mol Biol Evol 2011, 28:2893-2904.

11. Rapp RA, Wendel JF: Epigenetics and plant evolution. New Phytol 2005, 168:81-91.

12. Rodin S, Riggs A: Epigenetic silencing may aid evolution by gene duplication. J Mol Evol 2003, 56:718-729.

13. Rodin SN, Parkhomchuk DV: Position-associated GC asymmetry of gene duplicates. J Mol Evol 2004, 59:372-384.

14. Rodin SN, Parkhomchuk DV, Rodin AS, Holmquist GP, Riggs AD: Repositioning-dependent fate of duplicate genes. DNA Cell Biol 2005, 24:529-542.

15. Zheng D: Asymmetric histone modifications between the original and derived loci of human segmental duplications. Genome Biol 2008, 9:R105.

16. Cedar $\mathrm{H}$, Bergman $\mathrm{Y}$ : Linking DNA methylation and histone modification: patterns and paradigms. Nat Rev Genet 2009, 10:295-304.

17. Gilbert N, Thomson I, Boyle S, Allan J, Ramsahoye B, Bickmore WA: DNA methylation affects nuclear organization, histone modifications, and linker histone binding but not chromatin compaction. J Cell Biol 2007, 177:401-411.

18. Binz T, D'Mello N, Horgen PA: A comparison of DNA methylation levels in selected isolates of higher fungi. Mycologia 1998, 90:785-790.

19. Lim LP, Glasner ME, Yekta S, Burge CB, Bartel DP: Vertebrate microRNA genes. Science 2003, 299:1540.

20. Jenuwein T, Allis CD: Translating the histone code. Science 2001, 293: 1074-1080.

21. Strahl $B D, A$ llis $C D$ : The language of covalent histone modifications. Nature 2000, 403:41-45.

22. Kouzarides T: Chromatin modifications and their function. Cell 2007, 128:693-705.

23. Li B, Carey M, Workman JL: The role of chromatin during transcription. Cell 2007, 128:707-719.
24. van Steensel B: Mapping of genetic and epigenetic regulatory networks using microarrays. Nat Genet 2005, 37:S18-24.

25. Millar CB, Grunstein M: Genome-wide patterns of histone modifications in yeast. Nat Rev Mol Cell Biol 2006, 7:657-666.

26. Kurdistani SK, Grunstein M: Histone acetylation and deacetylation in yeast. Nat Rev Mol Cell Biol 2003, 4:276-284.

27. Sterner DE, Grant PA, Roberts SM, Duggan L, Belotserkovskaya R, Pacella LA, Winston F, Workman $J$, Berger SL: Functional organization of the yeast SAGA complex: distinct components involved in structural integrity, nucleosome acetylation, and TATA-binding protein interaction. Mol Cell Biol 1999, 19:86-98.

28. Benoist C, Chambon P: In vivo sequence requirements of the SV40 early promoter region. Nature 1981, 290:304-310.

29. Basehoar AD, Zanton SJ, Pugh BF: Identification and distinct regulation of yeast TATA box-containing genes. Cell 2004, 116:699-709.

30. Li W-H, Yang J, Gu X: Expression divergence between duplicate genes. Trends Genet 2005, 21:602-607.

31. Tirosh I, Weinberger A, Carmi M, Barkai N: A genetic signature of interspecies variations in gene expression. Nat Genet 2006, 38:830-834.

32. Landry CR, Lemos B, Rifkin SA, Dickinson WJ, Hartl DL: Genetic properties influencing the evolvability of gene expression. Science 2007, 317:118-121.

33. Suganuma T, Workman JL: Crosstalk among Histone Modifications. Cell 2008, 135:604-607.

34. O'Connor TR, Wyrick JJ: ChromatinDB: a database of genome-wide histone modification patterns for Saccharomyces cerevisiae. Bioinformatics 2007, 23:1828-1830.

35. Spellman PT, Sherlock G, Zhang MQ, lyer VR, Anders K, Eisen MB, Brown PO, Botstein D, Futcher B: Comprehensive identification of cell cycleregulated genes of the yeast Saccharomyces cerevisiae by microarray hybridization. Mol Biol Cell 1998, 9:3273-3297.

36. Chu S, DeRisi J, Eisen M, Mulholland J, Botstein D, Brown PO, Herskowitz I: The transcriptional program of sporulation in budding yeast. Science 1998, 282:699-705.

37. Cho RJ, Campbell MJ, Winzeler EA, Steinmetz L, Conway A, Wodicka L, Wolfsberg TG, Gabrielian AE, Landsman D, Lockhart DJ, Davis RW: A genome-wide transcriptional analysis of the mitotic cell cycle. Mol Cell 1998, 2:65-73.

38. Steinfeld I, Shamir R, Kupiec M: A genome-wide analysis in Saccharomyces cerevisiae demonstrates the influence of chromatin modifiers on transcription. Nat Genet 2007, 39:303-309.

39. Hu Z, Killion PJ, lyer VR: Genetic reconstruction of a functional transcriptional regulatory network. Nat Genet 2007, 39:683-687.

40. Lee TI, Rinaldi NJ, Robert F, Odom DT, Bar-Joseph Z, Gerber GK, Hannett NM, Harbison CT, Thompson CM, Simon I, Zeitlinger J, Jennings EG, Murray HL, Cordon DB, Ren B, Wyrick JJ, Tagne JB, Volkert TL, Fraenkel E, Gifford DK, Young RA: Transcriptional regulatory networks in Saccharomyces cerevisiae. Science 2002, 298:799-804.

41. Harbison CT, Gordon DB, Lee TI, Rinaldi NJ, Macisaac KD, Danford TW, Hannett NM, Tagne J-B, Reynolds DB, Yoo J, Jennings EG, Zeitlinger J, Pokholok DK, Kellis M, Rolfe PA, Takusagawa KT, Lander ES, Gifford DK, Fraenkel E, Young RA: Transcriptional regulatory code of a eukaryotic genome. Nature 2004, 431:99-104.

42. Brem RB, Kruglyak L: The landscape of genetic complexity across 5,700 gene expression traits in yeast. Proc Natl Acad Sci U S A 2005, 102:1572-1577.

43. Choi JK, Kim Y-J: Epigenetic regulation and the variability of gene expression. Nat Genet 2008, 40:141-147.

44. Martin D, Brun C, Remy E, Mouren P, Thieffry D, Jacq B: GOToolBox: functional analysis of gene datasets based on Gene Ontology. Genome Biol 2004, 5:R101.

45. Gu Z, Cavalcanti A, Chen F-C, Bouman P, Li W-H: Extent of Gene Duplication in the Genomes of Drosophila, Nematode, and Yeast. Mol Biol Evol 2002, 19:256-262.

46. Yang Z, Bielawski JP: Statistical methods for detecting molecular adaptation. Trends Ecol Evol 2000, 15:496-503.

doi:10.1186/1471-2148-12-111

Cite this article as: Zou et al:: Histone modification pattern evolution after yeast gene duplication. BMC Evolutionary Biology 2012 12:111. 